Europhys. Lett., 63 (3), pp. 476-477 (2003)

Erratum

\title{
Vorticity quantum numbers for confined electrons
}

\author{
L. F. Chibotaru ${ }^{1}$, A. Ceulemans ${ }^{1}$, M. Lorenzini ${ }^{2}$ and V. V. Moshchalkov ${ }^{3}$ \\ 1 Afdeling Kwantumchemie, Katholieke Universiteit Leuven \\ Celestijnenlaan 200F, B-3001 Leuven, Belgium \\ 2 IMEC-Interuniversity Microelectronics Center - Kapeldreef 75 \\ B-3001 Leuven, Belgium \\ 3 Laboratorium voor Vaste-Stoffysica en Magnetisme, Katholieke Universiteit Leuven \\ Celestijnenlaan 200D, B-3001 Leuven, Belgium
}

(Europhys. Lett., 63 (2), pp. 159-165 (2003))

PACS. 03.65.-w - Quantum mechanics.

PACS. 03.65.Vf - Phases: geometric; dynamic or topological (quantum theory).

Due to a technical problem occurred in production, in figs. 1 and 2 the minus signs were missing. We publish here again the correct figures sincerely apologizing to the authors for the unpleasant inconvenience.

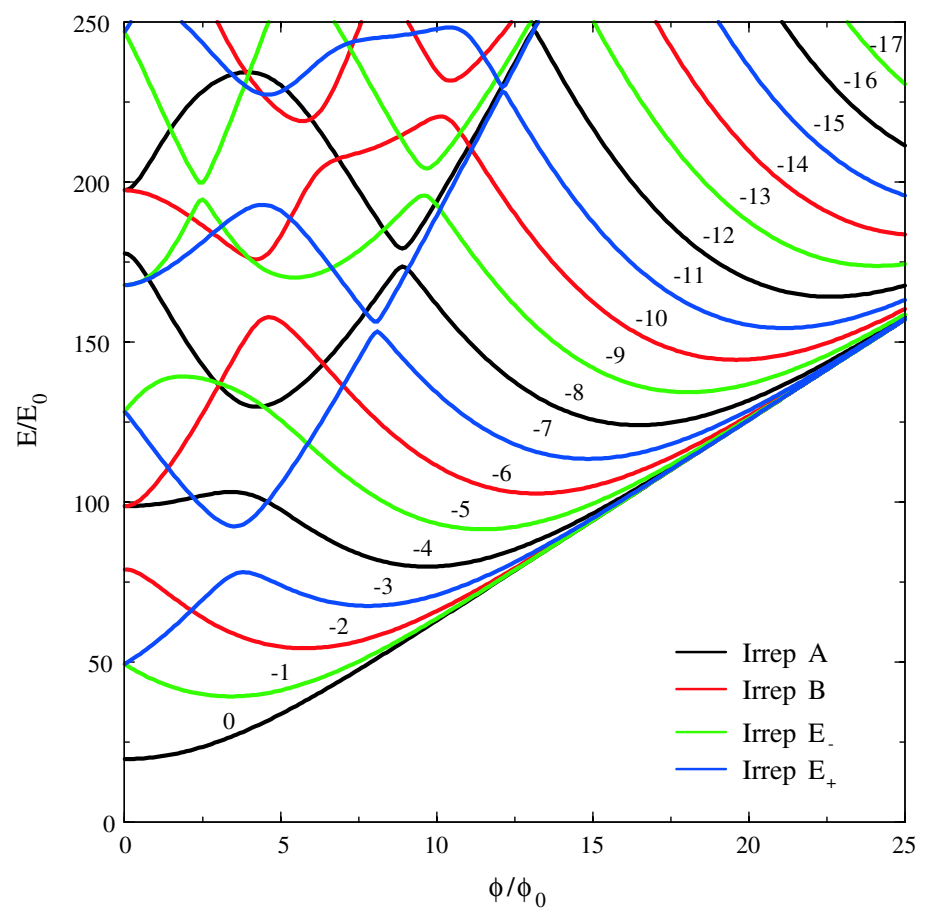

Fig. 1 - Energy spectrum of a negatively charged particle in a square in homogeneous magnetic field $H$ as a function of the applied flux $\phi=H S ; S$ is the area of the square, $\phi_{0}=h /|e| c$ is the normal flux quantum and $E_{0}=\hbar^{2} / 2 m S$.

(c) EDP Sciences 


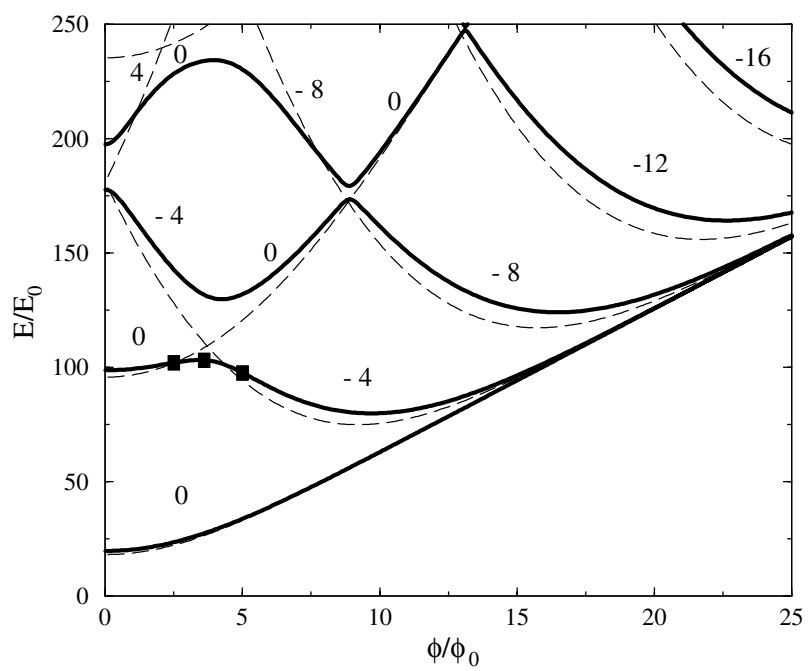

Fig. 2 - Low-energy region of the spectrum of eigenstates of symmetry $A$ (full lines) and the corresponding diagram of diabatic states levels (dashed lines). Vorticity numbers are indicated at each level. 